\title{
Commentary
}

\section{Mankind, Disease and Technology: A Cat, Mouse and Fiddle Game}

Srinivasan Govindaraj, MBBS, MPH*

Visiting Researcher, Ryerson University, Toronto, ON M5B 2K3, Canada

"Corresponding author

Srinivasan Govindaraj, MBBS, MPH

Visiting Researcher, Ryerson University, Toronto, ON M5B 2K3, Canada; Ph. + I-226-224-8645; E-mail: drgsrini20II@gmail.com

\section{Article information}

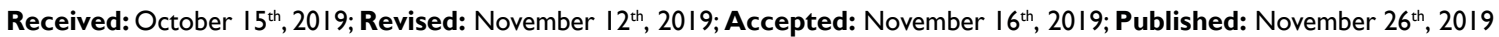

\section{Cite this article}

Govindaraj S. Mankind, disease and technology:A cat, mouse and fiddle game. Public Health Open J. 20 I9; 4(2): 56. doi: I0. I7| 40/PHOJ-4-I35

$\mathrm{M}$ ankind has been playing a cat and mouse game with diseases almost from the start of human civilization. Sometimes we have had a resounding victory, but sometimes disease has won, nearly wiping out populations in its path. Be it plague, polio, smallpox, cholera or acquired immunodeficiency syndrome (AIDS) all have left an indelible impression on humans. Just as in warfare, all these diseases have been controlled by essentially being one step ahead of them. Hence the fiddle or third factor has been technology, which could help us win this crucial time race.

Technology has enveloped all aspects of our life and it makes sense to make use of it in the field of health. Public health could be the greatest beneficiary, as new challenges continue to grow with climate change, resurgence of diseases as well as emergence of new ones, their spread being threatened by rapidly increasing air travel.

More specifically, the latest arms of technology in form of artificial intelligence (AI) or machine learning have thrown open new vistas for medical science. AI is the science of creating intelligent systems that help solve complex problems. Various potential applications include data analysis, fields of imaging, oncology, clinical decision support systems, drug research and software that uses AI in wearable technology such as Fitbits and smartwatches that helps to alert users after analyzing data.

One clear example of predictive analytics, is the use of complex data gathered through various means and processed using AI, being used to predict epidemic outbreaks. An example is the start-up AIME developing a software platform to predict dengue and Zika outbreaks. This then gives us the time to focus resources in places where it is needed and also contain it at the earliest, more importantly in resource constrained settings. Another use with AI is in analytics, where many of the radiology information systems (RIS) contain post-acquisition analysis modules that can spot pathology, and perform three dimensional (3D) reconstruction of standard 2D images. But caution has to be exercised with technology in healthcare, as there are unique problems unlike in any other field such as patient privacy, ethics and bias.

The National Health Service (NHS) in England is setting up a national AI laboratory to enhance care of patients and research. According to the Philips Future Health Index 2019, ${ }^{1}$ among US healthcare professionals about 33\% use AI-powered solutions in their practice or hospital, while countries like Germany (41\%) and China (85\%) surpass the US in the use of AI technologies among healthcare professionals. There is almost a global race among countries to gain leadership in AI and significant funding with investments running into billions of dollars in AI start-ups, with many focussed on healthcare. Hence, taking everything into consideration, even though the music from the fiddle is new, it is time to listen and embrace change, with adequate safeguards and cautious optimism.

\section{REFERENCE}

1. Philips Future Health Index 2019: Philips Future Health Index 2019 finds US among leaders in EMR use and AI spend, but overall digital health technology adoption mixed. Philips News Centre. Web site. https://www.usa.philips.com/a-w/about/ news/archive/standard/news/press/2019/20190717-philipsfuture-health-index-2019-finds-us-among-leaders.html. Accessed October 14, 2019. 